\title{
Computer Algebra Investigation of Known Primitive Triangle-Free Strongly Regular Graphs
}

\author{
Mikhail Klin and Matan Ziv-Av \\ Ben-Gurion University of the Negev \\ klin@cs.bgu.ac.il \\ matan@svgalib.org
}

\begin{abstract}
With the aid of computer algebra systems COCO and GAP with its packages we are investigating all seven known primitive triangle-free strongly regular graphs on 5, 10, 16, 50, 56, 77 and 100 vertices. These graphs are rank 3 graphs, having a rich automorphism group. The embeddings of each graph from this family to other ones are described, the automorphic equitable partitions are classified, all equitable partitions in the graphs on up to 50 vertices are enumerated. Basing on the reported computer aided results and using techniques of coherent configurations, a few new models of these graphs are suggested, which are relying on knowledge of just a small part of symmetries of a graph in consideration.
\end{abstract}

\section{Introduction}

This paper appears on the edge between computer algebra systems and algebraic graph theory (briefly AGT) and is mainly devoted to the computer aided investigation of the known primitive triangle free strongly regular graphs.

In fact, there are 7 such graphs, those on $5,10,16,50,56,77$ and 100 vertices. The largest one, denoted by $N L_{2}(10)$ is the universal graph for this family, in the sense that all seven graphs are induced subgraphs of $N L_{2}(10)$. We denote by $\mathfrak{F}$ the family of those 7 graphs.

The history of the discovery of the graphs from $\mathfrak{F}$, and in particular of $N L_{2}(10)$, is quite striking. Indeed, the graph $N L_{2}(10)$ was discovered twice: once in 1956 by Dale Mesner, and second time, independently, in 1968 by D. G. Higman and C. C. Sims. The details of this history are considered in [15, see also a very brief summary in Section 4 .

The graphs on 5, 10 and 50 vertices form a very significant subfamily, consisting of the known Moore graphs. One more possible member of this series (see e.g. [16]) would have 3250 vertices; its existence is an open problem of a great significance. Moore graphs and their natural generalizations are objects of much interest in extremal graph theory; they serve as the best examples of optimal network topologies. Discovery of a new primitive triangle free strongly regular graph would be a high level sensation in modern mathematics.

This text is oriented towards an interdisciplinary audience, though its kernel is strictly related to a concrete kind of application of computer algebra in AGT.

Section 2 provides in compact form the necessary background about strongly regular graphs, see [11, [5] for more details, while in section 3 we briefly introduce the tools of computer algebra used in AGT. The seven graphs from the family $\mathfrak{F}$ are presented in section 4

Our first main result is introduced in Section 5 full description of embeddings of (primitive and imprimitive) triangle free strongly regular graphs into the graphs in $\mathfrak{F}$. 
The next topic of our investigation is related to equitable partitions with a special emphasis on automorphic equitable partitions, see definitions in Section 6. The search strategy is described in Section 7, though much more details may be found in [28. We report the complete enumeration of automorphic equitable partitions and partial results for the general case.

Sections 810 reflect our efforts to understand a few of the computer aided results and to present them in a computer free form (completely, or at least relatively).

In Section 8 we deal with the graph $N L_{2}(10)$ and describe it in a sense locally, that is via so-called metric equitable partitions, obtained from the embeddings of a quadrangle and a graph $K_{3,3}$ without one edge (Atkinson configuration), with 4 and 8 cells respectively. The spectrum of the second partition contains all of the eigenvalues of $N L_{2}(10)$ (the case of full spectrum). Some modifications of the famous Robertson model (see 21]) appear in Section 9 . while in Section 10 we present a handful of models for some other graphs in $\mathfrak{F}$.

It is worthy to mention that for generations of mathematicians, the graphs in $\mathfrak{F}$ appear as a source of ongoing aesthetic admiration; see, especially the home page of Andries Brouwer $\underline{6}$. We believe that some of our models shed a new light on those graphs.

Last but not least, is that as a rule, the models we suggest rely on a relatively small subgroup of the group $\operatorname{Aut}(\Gamma)$, for $\Gamma \in \mathfrak{F}$. In this way we provide promising patterns for those researchers who wish in future to face a challenge: to try to construct new primitive triangle free strongly regular graphs, cf. Section 11 .

\section{Strongly regular graphs: a brief survey}

An undirected graph $\Gamma$ is called a strongly regular graph (SRG) with parameters $(v, k, \lambda, \mu)$ if it is a regular graph of order $v$ and valency $k$, and every pair of adjacent vertices has exactly $\lambda$ common neighbors, while every pair of non-adjacent vertices has exactly $\mu$ common neighbors. Sometimes we use an extended set of parameters, $(v, k, l, \lambda, \mu)$, where $l$ is the number of nonneighbors of a vertex, that is $l=v-k-1$.

If $A=A(\Gamma)$ is the adjacency matrix of an undirected graph $\Gamma$, then $\Gamma$ is strongly regular if and only if $A^{2}=k I+\lambda A+\mu(J-I-A)$. This implies that $(I, A, J-I-A)$ is the standard basis of a rank 3 homogeneous coherent algebra. In other words, $(\Delta, \Gamma, \bar{\Gamma})$ are the basic graphs of a rank 3 symmetric association scheme (here $\bar{\Gamma}$ is the complement of $\Gamma$, while $\Delta$ contains all the loops). The adjacency matrix of a strongly regular graph has exactly 3 distinct eigenvalues. For a strongly regular graph we denote by:

- $r>s$, the two eigenvalues of $A(\Gamma)$ different from $k ; r$ is always positive, while $s$ is always negative;

- $f, g$, the multiplicity of the eigenvalues $r, s$ respectively.

A formula for $f$ and $g$ is given by $g, f=\frac{1}{2}\left[(v-1) \pm \frac{2 k+(v-1)(\lambda-\mu)}{\sqrt{(\lambda-\mu)^{2}+4(k-\mu)}}\right]$.

A quadruple of parameters $(v, k, \lambda, \mu)$ for which $f$ and $g$ as given by the preceding formulas are positive integers is called a feasible set of parameters. See [6] for a list of feasible parameter sets with some information about known graphs for some of the sets.

A strongly regular graph $\Gamma$ is called primitive if both $\Gamma$ and its complement $\bar{\Gamma}$ are connected. This is equivalent to primitivity of the related association scheme $(\Delta, \Gamma, \bar{\Gamma})$. 


\section{Computer algebra tools in AGT}

During the last two decades the significance of the use of computer algebra systems in AGT increased drastically. Such systems are exploited in the search for new combinatorial objects, the enumeration of objects with prescribed parameters and the understanding of algebraic and structural properties of a given combinatorial object $\Gamma$ (such as the automorphism group $A u t(\Gamma)$, its action and orbits on the ingredients of $\Gamma$, enumeration of substructures of $\Gamma$, embeddings of $\Gamma$ into larger structures, etc.)

An ideal case is when the computer aided understanding of an object $\Gamma$ is followed by further theoretical generalizations. The foremost goal of this paper is to share with the reader many new interesting properties of the graphs in the family $\mathfrak{F}$ in order to promote the search for new primitive tfSRGs.

Below are the main tools we use.

- COCO is a set of programs for dealing with coherent configurations, including construction, enumeration of subschemes, and calculation of automorphism groups.

Developed in 1990-2, Moscow, USSR, mainly by Faradžev and Klin [8], [9].

- WL-stabilization - Weisfeiler-Leman stabilization is an efficient algorithm for calculating coherent closure of a given matrix (see [25], 3]). Two implementations of the WL-stabilization are available (see [2]).

- GAP an acronym for "Groups, Algorithms and Programming", is a system for computation in discrete abstract algebra [10], 22]. It supports easy addition of extensions (packages, in gap nomenclature), that are written in the GAP programming language which can add new features to the GAP system.

One such package, GRAPE [23], is designed for construction and analysis of finite graphs. GRAPE itself is dependent on an external program, nauty [18] in order to calculate the automorphism group of a graph. Another package is DESIGN, used for construction and examination of block designs.

- COCO v.2 - The COCO v.2 initiative aims to re-implement the algorithms in COCO, WL-stabilization and DISCRETA as a GAP package. In addition, the new package should essentially extend abilities of current version basing on new theoretical results obtained since the original COCO package was written.

We refer to 14 for a more detailed discussion of the ways in which computer algebra systems are used in AGT for the purpose of experimentation and further theoretical generalizations.

\section{The seven known primitive triangle-free strongly regu- lar graphs}

A graph $\Gamma$ is called triangle free if it admits no triangles, that is cliques of size 3 . If $\Gamma$ is also a strongly regular graph then it is called a triangle free strongly regular graph (tfSRG for short). A graph is triangle free if any two neighbors have no common neighbors, therefore a tfSRG is an SRG with $\lambda=0$.

The 7 known primitive tfSRGs, with orders from 5 to 100 vertices are:

1. Pentagon with parameters $(5,2,0,1)$. Its automorphism group is $D_{5}$ of order 10 . 


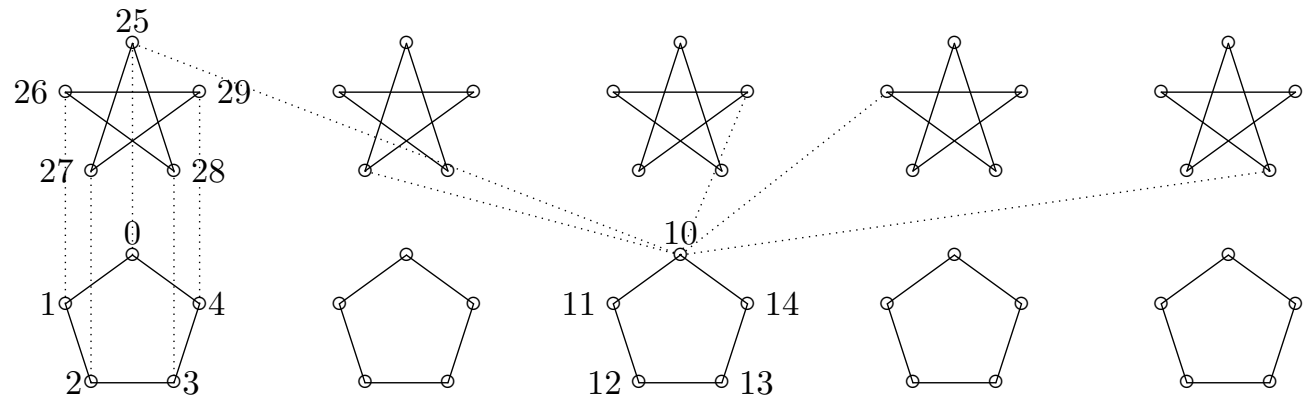

Figure 1: Hoffman-Singleton graph, Robertson model

2. Petersen graph with parameters $(10,3,0,1)$. Its automorphism group is isomorphic to $S_{5}$ of order 120 . A simple model has as vertices 2 -subsets of a set of size 5 , with two vertices adjacent if the subsets are disjoint.

3. Clebsch graph with parameters $(16,5,0,2)$. Usually denoted by $\square_{5}$. Its automorphism group is isomorphic to $\left(S_{5} 2 S_{2}\right)^{\text {pos }}$ of order 1920. A simple model is 4-dimensional cube $Q_{4}$ together with long diagonals, or Cayley graph: $C A Y\left(E_{2^{4}},\{0001,0010,0100,1000,1111\}\right)$.

4. Hoffman-Singleton graph $(\mathrm{HoSi})$ with parameters $(50,7,0,1)$. Its automorphism group is isomorphic to $P \Sigma U\left(3,5^{2}\right)$ of order 252000 (4 4$)$. The simplest model is Robertson model ([21]): 5 pentagons marked $P_{0}, \ldots, P_{4}$ and 5 pentagrams marked $Q_{0}, \ldots, Q_{4}$ with vertex $i$ of $P_{j}$ joined to vertex $i+j k(\bmod 5)$ of $Q_{k}$.

5. Gewirtz (or Sims-Gewirtz) graph with parameters $(56,10,0,2)$. Its automorphism group of order 80640 is a non split extension of $P S L_{3}(4)$ by $E_{2^{2}}$. A simple model is as the induced subgraph of $N L_{2}(10)$ on the common non-neighbors of two adjacent vertices.

6. Mesner graph with parameters $(77,16,0,4)$. The automorphism group is of order 887040 and is isomorphic to the stabilizer of a point in the automorphism group of $N L_{2}(10)$. One simple model is: induced subgraph of $N L_{2}(10)$ on non-neighbors of a vertex.

7. $N L_{2}(10)$ with parameters $(100,22,0,6)$, also known as the Higman-Sims graph. Its automorphism group contains Higman-Sims sporadic simple group as a subgroup of index 2 . We refer to [15] for a detailed presentation of the original construction of this graph by Dale Mesner, as it appeared on [19], 20].

Recall that the graph $N L_{2}(10)$ on 100 vertices was constructed twice: by Dale Mesner in his Thesis in 1956 [19] and later on by Higman and Sims in 1968, when they discovered a new sporadic simple group. Moreover, in 1964 Mesner proved its uniqueness. We denote this graph by $N L_{2}(10)$, following the parametrization for a family of putative graphs, introduced by Mesner in 20. In [15] the history of this discovery, as well as of related events is discussed with many details. A new wave of interest in tfSRGs, stimulated by [15], was especially productive for us. It became clear that this is the time to arrange a serious comprehensive investigation of diverse structural properties of the graphs in the family $\mathfrak{F}$. The goal is to attract the attention of researchers to such an information in order to provide new ideas how efforts for the search of new tfSRGs should be arranged. We also wish to share with the reader some surprising new features of the graphs in the family $\mathfrak{F}$, observed with the aid of a computer. 


\section{Embeddings of tfSRGs into known primitive tfSRGs}

Usually in graph theory a graph $\Delta=\left(V^{\prime}, E^{\prime}\right)$ is called a subgraph of a graph $\Gamma=(V, E)$ if $V^{\prime} \subseteq V$ and $E^{\prime} \subseteq E$. Of special interest are induced subgraphs, in this case $E^{\prime}=E \cap\left\{\begin{array}{c}V^{\prime} \\ 2\end{array}\right\}$, where $\left\{\begin{array}{l}X \\ 2\end{array}\right\}$ is the set of all 2-subsets of $X$.

Proposition 1. A subgraph $\Delta$ of diameter 2 of a graph $\Gamma$ with no triangles is an induced subgraph.

Proof. Assume that $\Delta$ is not induced, then there exists an edge $\{u, w\} \in E,\{u, w\} \notin E^{\prime}$ with $u, w \in V^{\prime}$. Since the diameter of $\Delta$ is 2 , there exist a $v$ which is adjacent in $\Delta$ (and therefore in $\Gamma$ ) to $u, w$, thus $\{u, v, w\}$ is a triangle in $\Gamma$, which is a contradiction.

The enumeration of all tfSRGs inside of known tfSRGs was the first serious computational challenge in this project. The results of its complete solution are discussed below.

A non-empty imprimitive tfSRG is either a complete bipartite graph $\left(K_{l, l} \cong \overline{2 \circ K_{l}}\right)$, or a graph $s \circ K_{2}$ consisting of $s$ edges with no common vertices. Note that $K_{2,2}$ is simply a quadrangle.

Lemma 2. Graph $N L_{2}(10)$ does not contain subgraph $K_{3,3}$.

Proof. Originally, we obtained this result with the aid of a computer. In fact, easy proof follows from the non-edge decomposition of $\Gamma=N L_{2}$ (10) (see e.g. [15]), without any assumptions about its uniqueness. Indeed, let $\Delta$ be a subgraph of $\Gamma$, which is isomorphic to $K_{3,3}$. Assume that two bipartite parts of $\Delta$ consist of subsets $\{a, b, c\},\{d, e, f\}$ of vertices. Consider now non-edge decomposition of $\Gamma$ with respect to $\{a, b\}$, then $c$ belongs to the cell of size 60 , while $d, e, f$ belong to the cell of size 6 , thus $c$ has just $g=2$ neighbors in the latter cell, a contradiction with the valency of $c$, equal to 3 in $\Delta$.

As a corollary, we obtain that every known primitive tfSRG does not contain $K_{3,3}$, and moreover does not contain $K_{l, l}$, when $l \geq 3$.

Another simple theoretical result (see e.g. 28) claims that the number of quadrangles in an SRG with parameters $(v, k, l, \lambda, \mu)$ is equal to $\frac{\frac{v k}{2}\left(\begin{array}{l}\lambda \\ 2\end{array}\right)+\frac{v l}{2}\left(\begin{array}{c}\mu \\ 2\end{array}\right)}{2}$. When $\lambda=0$ this reduces to $\frac{v l \mu(\mu-1)}{8}$. Similar formula may be obtained for the number of $2 \circ K_{2}$. The numbers of imprimitive tfSRGs inside of graphs in $\mathfrak{F}$ are given in Table 1 in the supplement, while the number of orbits of such embeddings (with respect to $A u t(\Gamma)$ for $\Gamma \in \mathfrak{F}$ ) are given in Table 2 .

Note that there is no induced subgraph of $N L_{2}(10)$, isomorphic to $12 \circ K_{2}$, so both tables in Supplement A end with $s=11$ for $s \circ K_{2}$.

Similar computer results are presented in Tables 3 and 4 (Supplement A for the embeddings of a graph from the family $\mathfrak{F}$ into a larger graph in $\mathfrak{F}$.

Here the final picture is less sophisticated. There are just two cases where for pair of graphs $X, Y \in \mathfrak{F}$ there exist more than one orbit of embeddings (up to action of $A u t(Y)$ ) of $X$ into $Y$. Namely, in these terms, there are 9 embeddings of Petersen graph into Mesner graph and 5 embeddings into $N L_{2}(10)$. Computer free understanding of these embeddings remains a quite interesting task for future investigations of the links between the graphs in the family $\mathfrak{F}$.

In all other cases there exists (up to isomorphism) at most one embedding for considered pair $(X, Y)$. For most pairs there is a reasonably clear explanation in literature, such as two HoSi inside $N L_{2}(10)$, see e.g. [12, as well as Robertson model of HoSi [21, which explains positions in it for both pentagon and Petersen graph.

Beside this there are two more embeddings, which are not immediately evident. 
The first pair is the embedding of the Petersen graph into Gewirtz graph. In fact, in this embedding the Petersen graph appears as a subgraph of the second constituent of the Gewirtz graph, which in turn is a graph $\Delta$ of valency 8 on 45 vertices. It turns out that $\operatorname{Aut}(\Delta) \cong \operatorname{Aut}\left(S_{6}\right)$. This graph $\Delta$ generates a non-Schurian association scheme with three classes, see e.g. [24]; 13], example $W_{38}$. The graph $\Delta$ may be also described as the distance 2 graph of the distance transitive graph of diameter 4 and valency 4 on 45 vertices, a.k.a generalized octagon $G O(2,1)$, a.k.a line graph of Tutte's 8-cage (see 5 for more details). In this context, the task of explanation of the embedding of Petersen graph into $\Delta$ seems to be a nice exercise in AGT, though out of the framework of this presentation.

The second exceptional pair is the embedding of the Clebsch graph $\square_{5}$ into $N L_{2}(10)$. This pair is of a definite independent interest and thus is considered separately in Section 10.

Finally we mention that the classification of cocliques in graphs from $\mathfrak{F}$ may be regarded as a degenerate case of subject in this section. Indeed, empty graph is a particular case of an imprimitive tfSRG. We however disregard this problem, referring to information presented on the home page of Andries Brouwer [6].

\section{Equitable partitions}

Let $\Gamma=(V, E)$ be a graph. A partition $\tau$ of the vertex set $V, \tau=\left\{V_{1}, \ldots, V_{s}\right\}$ is called equitable partition (briefly, EP) if for $i, j \in\{1, \ldots, s\}$, the numbers $\left|\Gamma(v) \cap V_{j}\right|$ are equal for all $v \in V_{i}$. Here $\Gamma(v)=\{u \in V \mid\{u, v\} \in E\}$. Usually an EP $\tau$ is accompanied by intersection diagram, which represents a kind of quotient graph, $\Gamma / \tau$, on which all intersection numbers are depicted. Many such diagrams appear in [5. The quotient graph $\Gamma / \tau$ is, in fact, a multigraph. Its (collapsed) adjacency matrix $B$ consists of all intersection numbers.

Obviously, entries of $B$ are non-negative integers, and for a regular graph $\Gamma$ of valency $k$ the sum of each row in $B$ is $k$.

If $H$ is a subgroup of $A u t(\Gamma)$, then the set of orbits of $H$ is an EP of $\Gamma$. Such an EP is called automorphic (briefly AEP).

Proposition 3 ([1]). Let $\Gamma$ be a graph, $A=A(\Gamma)$ the adjacency matrix of $\Gamma$. If a partition $\tau$ is $E P$ of $\Gamma$ with matrix $B$ then the characteristic polynomial of $B$ divides the characteristic polynomial of $A$.

In fact, there are more necessary conditions for a prescribed matrix $B$ to be the adjacency matrix of a suitable EP of $\Gamma$, which are formulated in terms of spectral graph theory, see e.g. 7. They create a solid background for a clever search of potential EPs in a given graph $\Gamma$.

\section{$7 \quad$ Search for equitable partitions}

In this project we distinguished two alternative problems for the complete search of EPs in graphs of $\mathfrak{F}$.

First problem is to enumerate all automorphic EPs. Here we strongly rely on the level of group theoretical "intellect" of GAP. Indeed, for groups of relatively small order (say up to a few thousands) GAP allows to establish efficiently the structure of a considered group, as well as the lattice of its subgroups.

However, for four graphs in $\mathfrak{F}$ the group $G=A u t(\Gamma)$ has larger order, thus with the growing of $|G|$ extra theoretical input is required. For example, in case of $G=\operatorname{Aut}\left(N L_{2}(10)\right)$ we were using information from the website of the Atlas of Finite Group Representations ([26]) about 
the maximal subgroups of $G$ (this information goes back to [17]). This knowledge, together with ad hoc tricks inside of GAP made it possible to describe all subgroups of $G$ up to equivalency classes with respect to EPs. The fact that all groups $G=\operatorname{Aut}(\Gamma), \Gamma \in \mathfrak{F}$, are subgroups of $\operatorname{Aut}\left(N L_{2}(10)\right)$ was also quite beneficial.

Finally, we successfully enumerated all automorphic EPs for all $\Gamma \in \mathfrak{F}$.

The second problem is much more sophisticated: to enumerate all EPs for all graphs in $\mathfrak{F}$.

For the smallest three graphs in $\mathfrak{F}$ the results were achieved via simple brute force, see corresponding tables in Supplement B.

For HoSi we used a few complementary strategies in the search for all EPs. First, we attacked partitions with "large" number of cells, say $s>5$. Here we introduced extra parameter: the size of the smallest cell, which varies from 1 to 9 . Each such case was considered separately.

On the second stage, we step by step enumerated cases $2 \leq s \leq 5$, by enumerating possible collapsed adjacency matrices for each case, and for any such matrix, enumerating all EPs.

The description of our computer activities together with detailed discussion of the results is presented in 28. An extra advantage of the enumeration in HoSi is that it serves as a kind of a pleasant interaction of a human and a computer. Indeed, in spite of the fact that the main part of computation was fulfilled by GAP, a human can follow search, explain its logic and step by step to comprehend all ongoing results.

We admit however that the extra advantage of $\mathrm{HoSi}$, is that here parameter $\mu$ takes its smallest possible value of 1 (for a primitive SRG). As soon as $\mu>1$ and $v$ is growing, the problem is becoming essentially more difficult.

This is why already for the Gewirtz graph, with $v=56$ we only succeeded in enumeration of all EPs for which the stabilizer has order at least 2. There are 688 such EPs. The enumeration of the rigid EPs cannot be completed by a computer in a reasonable time.

The full enumeration of all EPs for the largest two graphs in $\mathfrak{F}$ (on 77 and 100 vertices) at this stage looks intractable. Only modest particular cases may be proceeded efficiently.

We refer to Supplement B for Tables 5,6 and 7, which summarize our activities for both considered problems, see 28] for more information.

As was mentioned in the introduction, one of the main goals of this project is to detect a number of "nice" EPs, which may serve as a training model for future search of new primitive tfSRGs. At this stage this kind of job only partially relies on the support from computer, finally a human's insight still turns out to be very significant. Below we report about interesting interpretations created by us for a handful of detected EPs.

\section{Quadrangle and Atkinson EPs of $N L_{2}(10)$}

Recall that $\Gamma=N L_{2}(10)$ has $\frac{100 \cdot 77 \cdot 6 \cdot 5}{8}=28875$ quadrangles. This is a simple result, obtained on a combinatorial level without any knowledge of structure of $\Gamma$.

Provided that $\Gamma$ and $G=\operatorname{Aut}(\Gamma)$ are known, we easily obtain (with the use of a computer) that all quadrangles in $\Gamma$ form one orbit under $G$ and stabilizer $H$ of a quadrangle in $G$ has order 3072 , is isomorphic as abstract group to $\left.\left.\left.\left.\left.\left(\mathbb{Z}_{4} \times \mathbb{Z}_{2}\right) \cdot\left(\mathbb{Z}_{4} \times \mathbb{Z}_{2}\right)\right): \mathbb{Z}_{2}\right): \mathbb{Z}_{2}\right): \mathbb{Z}_{3}\right): \mathbb{Z}_{2}\right): \mathbb{Z}_{2}$ and has in action on $V(\Gamma)$ four orbits of lengths 4, 8, 24 and 64 .

Thus we may consider an automorphic EP of size $s=4$. There is sense to try again combinatorial arguments in order to determine all invariants of this EP without any prior knowledge of $\Gamma$ and its group. Fortunately, this turns out to be possible.

Proposition 4. Let $\tau$ be a metric decomposition with respect to a prescribed quadrangle $Q$ inside of $\Gamma$. Then $\tau$ is $E P, s=4$, sizes of cells are 4, 8, 24, 64 and (with respect to this 
ordering of cells), $B=\left(\begin{array}{cccc}2 & 4 & 0 & 16 \\ 2 & 0 & 12 & 8 \\ 0 & 4 & 2 & 16 \\ 1 & 1 & 6 & 14\end{array}\right)$.

See proof in 28 .

In principle, next stage should be to describe a model of $N L_{2}(10)$ in terms of the group $H$ of order 3072 or one of its subgroups which is transitive on all cells of the quadrangle EP. Nevertheless, in this text we are avoiding this task. Instead of, we prefer to split the quadrangle EP to one with larger number of cells and to proceed with that new one.

A note 1 was published before the announcement of CFSG, The author considered 13 parameter sets for tfSRGs, which may be also rank 3 graphs, with the number $v$ of vertices where $100<v \leq 1000$ and proved that such graphs can not be constructed with the aid of known 2-transitive permutation groups. Part of exposed details is related to consideration of a configuration $\mathfrak{A}$ (graph $K_{3,3}$ minus one edge) in putative graph.

We got impression that [1] is overseen through a generation of mathematicians and it might be helpful to analyze existence of $\mathfrak{A}$ (we call it Atkinson configuration) in known tfSRGs.

It turns out that $\mathfrak{A}$ appears only in graphs on 77 and 100 vertices. Here we analyze its position in $\Gamma=N L_{2}(10)$.

Let us fix at $\mathfrak{A}$ a quadrangle, for example with the vertices $\{a, b, c, f\}$. Then two extra vertices have two neighbors in the selected quadrangle, namely ends of two non-edges respectively.

Now we embed $\mathfrak{A}$ into the considered above quadrangle decomposition splitting in it cell of size 8 into two cells of size 2 (exactly our $\{d, e\}$ ) and the remainder. This immediately implies split of the cell of size 64 into two cells of sizes 16 and 48 . The cell of size 24 is forced to be split into subcells of sizes $12,6,6$, depending on the number of neighbors in $\{d, e\}$ being 1,2 and 0 , respectively.

A more careful analysis (see [28) shows that we are still getting an EP.

Proposition 5. A metrical decomposition $\tau(\mathfrak{A})$ of a tfSRG with the parameters of $N L_{2}(10)$ with respect to configuration $\mathfrak{A}$ is $E P$ with $s=8$ and cells of sizes 4, 2, 16, 48, 6, 12, 6, 6. It has collapsed matrix $B$ as follows, with $\operatorname{Spec}(B)=\left\{22,2^{5},(-8)^{2}\right\}$.

Originally, the proof was obtained with the aid of computer analysis of $\Gamma=N L_{2}(10)$. Later on an outline of a similar proof as for quadrangle configuration was again obtained without prior knowledge of $\Gamma$.

Further consideration of $\tau(\mathfrak{A})$ was fulfilled inside of known graph $\Gamma$ and its group $A u t(\Gamma)$. It turns out that $H=\operatorname{Aut}(\tau(\mathfrak{A})) \cong D_{4} \times S_{4}$ is a group of order 192. $H$ has exactly 8 orbits on the set $V(\Gamma)$, thus $\mathfrak{A}$ is an automorphic EP.

Describing further stabilizers of $H$ on each of the 8

$$
B=\left(\begin{array}{cccccccc}
2 & 1 & 4 & 12 & 3 & 0 & 0 & 0 \\
2 & 0 & 8 & 0 & 0 & 6 & 6 & 0 \\
1 & 1 & 2 & 12 & 0 & 3 & 0 & 3 \\
1 & 0 & 4 & 10 & 1 & 3 & 2 & 1 \\
2 & 0 & 0 & 8 & 0 & 6 & 2 & 4 \\
0 & 1 & 4 & 12 & 3 & 2 & 0 & 0 \\
0 & 2 & 0 & 16 & 2 & 0 & 0 & 2 \\
0 & 0 & 8 & 8 & 4 & 0 & 2 & 0
\end{array}\right)
$$
orbits we elaborated the following ad hoc model of $\Gamma$.

We start from the auxiliary graph $\Delta$ depicted in Figure 2

We identify elements of each orbit in $\tau(\mathfrak{A})$ with suitable sets of structures, described with the aid of $\Delta$. In fact, each set of structures appears as orbit of action of the group $H$. Thus it is enough to describe a representative of each orbit.

For example, the elements in the cell of size 16 are the vertices of $\Delta$, while the elements of the cell of size 4 are the four columns of $\Delta$. The most tricky to find was a representation of the 7 th cell, of size 6 . 
We think that this piecewise model of $\Gamma$, appearing from a reasonably small solvable group of order 192 may serve as an optimistic message for those researchers who are hunting for new tfSRGs, see also comments in Section 11 .

\section{Some modifications of Robertson model}

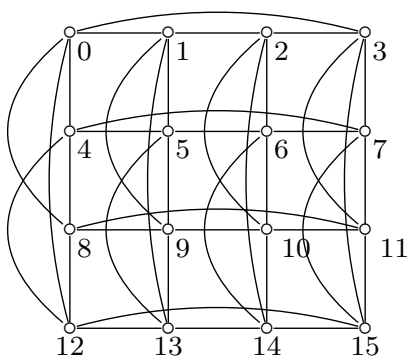

The Robertson model of the Hoffman-Singleton graph (see Figure 11) partitions the vertices into five pentagons and five pentagrams, and describes a simple arithmetic rule for the edges connecting pentagons and pentagrams (see Section 44. Let $p_{i j}\left(q_{i j}\right)$ be vertex $j$ of pentagon (pentagram) $i$.

Since $N L_{2}(10)$ has an EP to two cells of size 50, where the induced graph on each cell is isomorphic to HoSi, this model can be extended to a model of $N L_{2}(10)$.

In both cases we get an EP with a collapsed adjacency matrix that has a transitive automorphism group (a permutation $g \in S_{n}$ is an automorphism of a matrix $M \in \mathbb{F}^{n \times n}$ if the permutation matrix $M_{g}$ commutes with $M$ ). We are therefore interested in equitable partitions with this property.

In the list of equitable partitions of HoSi we find another such partition with 10 cells of size five. The collapsed adjacency matrix of this partition is $M$. The spectrum of $M$ is $\left\{7,2^{4},(-3)^{5}\right\}$, so it has the full spectrum of HoSi.

The cells of this partition can be described in the Robertson model of HoSi: for each $i \in[1,5]$, we construct two cells: $\left\{p_{1,1+i}, p_{2,1+i}, p_{3,2+i}, p_{4,4+i}, p_{5,2+i}\right\}$, $\left\{q_{1,1+i}, q_{2,1+i}, q_{3,5+i}, q_{4,3+i}, q_{5,5+i}\right\}$.

If we consider this matrix $M$ as the adjacency matrix of a color graph, then the basic graph for the color 1 is the Petersen graph, while the basic graph for the color 2 is a cycle of length 10. For comparison, acting similarly with the Robertson partition we get a graph $K_{5,5}$.

The stabilizer of the (ordered) partition in

$$
M=\left(\begin{array}{llllllllll}
0 & 2 & 2 & 0 & 0 & 0 & 0 & 1 & 1 & 1 \\
2 & 0 & 1 & 0 & 1 & 2 & 0 & 0 & 1 & 0 \\
2 & 1 & 0 & 2 & 0 & 0 & 1 & 0 & 0 & 1 \\
0 & 0 & 2 & 0 & 1 & 1 & 2 & 0 & 0 & 1 \\
0 & 1 & 0 & 1 & 0 & 2 & 0 & 1 & 0 & 2 \\
0 & 2 & 0 & 1 & 2 & 0 & 1 & 0 & 1 & 0 \\
0 & 0 & 1 & 2 & 0 & 1 & 0 & 1 & 2 & 0 \\
1 & 0 & 0 & 0 & 1 & 0 & 1 & 0 & 2 & 2 \\
1 & 1 & 0 & 0 & 0 & 1 & 2 & 2 & 0 & 0 \\
1 & 0 & 1 & 1 & 2 & 0 & 0 & 2 & 0 & 0
\end{array}\right)
$$
Aut $(\mathrm{HoSi})$ is isomorphic to the dihedral group $D_{5}$ of order 10, as is the automorphism group of $M$.

The coherent closure of the partition (more specifically, of the graph with its vertices colored according to the partition) is a Schurian coherent configuration of rank 300, with the stabilizer of the partition as its automorphism group.

This EP of HoSi can be extended to an EP of $N L_{2}(10)$, of 20 independent sets of size 5 . In this case, the matrix has spectrum $\left\{22,2^{13},(-8)^{6}\right\}$, so this EP has full spectrum as well.

The automorphism group of this matrix has order 240 and is isomorphic to $\mathbb{Z}_{2} \times S_{5}$, while the stabilizer of the ordered partition is again $D_{5}$ of order 10 .

The coherent closure of the partition is a Schurian coherent configuration of rank 1200, with the stabilizer of the partition as its automorphism group. 


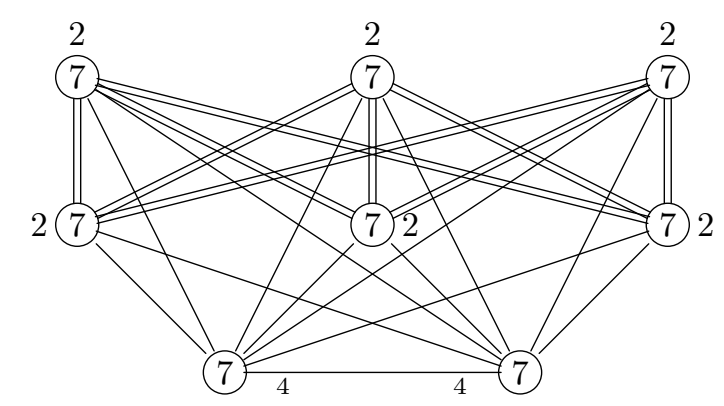

Figure 3: AEP of Gewirtz graph by a semiregular subgroup of order 7

\section{A few more models}

The calculated data, both about embeddings of tfSRGs inside tfSRGs and about EPs can be used to present new models and constructions for tfSRGs. Here we offer a few examples, without going into a lot of details:

Example 1 (Two Wagner graphs in Clebsch graph). Wagner graph, also known as Möbius ladder of order 8 , denoted by $M_{8}$ is a cubic graph on 8 vertices. A simple model for it is a $\operatorname{Cay}\left(\mathbb{Z}_{8},\{1,-1,4\}\right)$. The spectrum is $\left\{3,1^{2},-1\right\}$ and Aut $\left(M_{8}\right)=D_{8}$.

In one of the three EPs of $\square_{5}$ into two cells of size 8, the induced subgraph on each cell is the Wagner graph. The stabilizer of ordered partition inside Aut $\left(\square_{5}\right)$ is $D_{8}$.

Example 2 (Non-automorphic EP of HoSi). We can construct an EP of HoSi into two cells by taking one of the cells to be the 20 vertices of two Petersen graphs (any two pentagons and two pentagrams in Robertson model). The stabilizer of this partition is $D_{10}$ of order 20, so it is not automorphic.

Example 3 (Inside Gewirtz graph: six 7-cycles). There is one automorphic partition of Gewirtz graph into 8 cells of size 7 . The induced graph on 6 of the cells is a cycle of length 7, while the induced graph on the other two cells has no edges.

From the intersection diagram (Figure 3) we see that this partition can be merged to an equitable partition with 2 cells of sizes 42 and 14 with adjacency matrix $\left(\begin{array}{ll}8 & 2 \\ 6 & 4\end{array}\right)$.

The induced graph on the cell of size 42 of valency 8 is a coherent graph. Its coherent closure is a rank 6 imprimitive non-Schurian association scheme. This graph is not a rational graph, with spectrum $\left\{8,2^{22},(-1)^{7},(1+\sqrt{2})^{6},(1-\sqrt{2})^{6}\right\}$.

The induced graph on the cell of size 14 is a bipartite graph with two parts of size 7 . It is the co-Heawood graph, that is the bipartite complement to the Heawood graph, the Levi graph of Fano plane. This is by necessity true, since a regular bipartite graph of valency 4 and order 14 in which every vertex belongs to exactly one quadrangle is the co-Heawood graph.

A. Brouwer ([G]) notes that this co-Heawood graph is a subgraph of the Gewirtz graph.

Example 4 (Gewirtz graph inside of Mesner graph through the group $\mathbb{Z}_{7}$ ). We start with a semiregular subgroup $\mathbb{Z}_{7}$ of order 7 , this time inside of the automorphism group of Mesner graph. We get an AEP with 11 cells of size 7; the collapsed matrix $N$ of order 11 is presented in [28]. It turns out that Aut $(N) \cong S_{2} 2 S_{3}$ (the wreath product) is a group of order 72 with orbits of sizes $6,2,1^{3}$. There are three non-equivalent possibilities to merge from this AEP a 
non-automorphic EP with 3 cells of sizes 14, 21, 42 and a collapsed matrix $\left(\begin{array}{ccc}4 & 6 & 6 \\ 4 & 0 & 12 \\ 2 & 6 & 8\end{array}\right)$, which has full spectrum.

Each of the cases provides also an EP for the Gewirtz graph with two cells of sizes 14 and 42. Thus, as a by product, we obtain other (cf. Example 3) embeddings of the co-Heawood graph into the Gewirtz graph. This example demonstrates "irregularities", which may cause interesting EPs, unpredictable from the first sight.

Example 5 (Clebsch graph inside $N L_{2}(10)$ ). Recall that up to the automorphism group of $\Gamma=N L_{2}(10)$, there is one embedding of $\square_{5}$ into $\Gamma$. The equitable partition resulting from this embedding allows us to describe another model for $N L_{2}(10)$.

We start from a group $H_{2}=\mathbb{Z}_{4} \times S_{4}$ of order 96, with orbits of lengths 16, 16, 48, 12, 8. The collapsed adjacency matrix of the corresponding automorphic equitable partition is $B^{\prime \prime}$, (shown below). Clearly, the induced graph on the first cell of the partition is isomorphic to $\square_{5}$.

In principle, one may try as in previous sections to reconstruct the corresponding matrix $B$ from scratch, not relying on knowledge of the graph $\Gamma$ and its group. A promising starting point would be justification of existence of a coclique of size 8 and its adjacency with the induced $\square_{5}$.

We, however, prefer to exploit at this stage another possibility. Namely, it turns out that the stabilizers of $\mathfrak{A}$ and $\square_{5}$ inside of $\Gamma$ have maximal possible intersection $K=\mathbb{Z}_{4} \times S_{3}$ of order 24. $K$ defines an AEP with 11 cells of sizes 2, 4, 4, 6, 6, 6, 12, 12, 12, 12, 24 and matrix $B^{\prime}$.

$$
B^{\prime}=\left(\begin{array}{ccccccccccc}
0 & 2 & 2 & 0 & 0 & 6 & 0 & 6 & 0 & 6 & 0 \\
1 & 2 & 1 & 3 & 0 & 0 & 3 & 3 & 3 & 0 & 6 \\
1 & 1 & 2 & 0 & 3 & 0 & 3 & 0 & 3 & 3 & 6 \\
0 & 2 & 0 & 0 & 4 & 2 & 2 & 0 & 2 & 6 & 4 \\
0 & 0 & 2 & 4 & 0 & 2 & 2 & 6 & 2 & 0 & 4 \\
2 & 0 & 0 & 2 & 2 & 0 & 4 & 0 & 4 & 0 & 8 \\
0 & 1 & 1 & 1 & 1 & 2 & 4 & 3 & 2 & 3 & 4 \\
1 & 1 & 0 & 0 & 3 & 0 & 3 & 2 & 3 & 3 & 6 \\
0 & 1 & 1 & 1 & 1 & 2 & 2 & 3 & 4 & 3 & 4 \\
1 & 0 & 1 & 3 & 0 & 0 & 3 & 3 & 3 & 2 & 6 \\
0 & 1 & 1 & 1 & 1 & 2 & 2 & 3 & 2 & 3 & 6
\end{array}\right) \quad B^{\prime \prime}=\left(\begin{array}{ccccc}
5 & 4 & 9 & 3 & 1 \\
4 & 2 & 12 & 0 & 4 \\
3 & 4 & 11 & 3 & 1 \\
4 & 0 & 12 & 2 & 4 \\
2 & 8 & 6 & 6 & 0
\end{array}\right)
$$

It is easy to recognize how $B^{\prime}$ is split from the matrix $B$ of the metric EP of the configuration A. Then we merge the $B^{\prime}$ into $B^{\prime \prime}$ :

The cell of size 48 is a merging of the last 3 cells, the cell of size 8 is merging of cell of sizes 2 and 6 (5th cell), while the cell of size 12 is a merging of two remaining cells of size 6 . Each cell of size 16 is a merging of cells of sizes 4 and 12.

Example 6 (Reconstruction of $N L_{2}(10)$ from a subgroup of order 2). Using information in the atlas [26] (or direct calculation using GAP), we see that $G=\operatorname{Aut}\left(N L_{2}(10)\right.$ ) contains one conjugacy class of involutions with no fixed points. From such an involution, $i$, we get an AEP into 50 cells of size 2. We investigated the quotient graph $\widetilde{\Gamma}$ of this AEP, and its collapsed adjacency matrix, denoted by $B$. The structure of $\widetilde{\Gamma}$ may be recovered by considering the centralizer $C_{G}(i)$. However, we prefer to rely on information of combinatorial nature. The graph $\widetilde{\Gamma}$ has a natural EP into cells of sizes 20 and 30 (representing edges and non-edges in $\left.N L_{2}(10)\right)$.

The induced (color) graph on 20 vertices is the graph $K_{10,10}$ from which a 1-factor is removed.

The induced graph on 30 vertices is the incidence graph of generalized quadrangle of order 2, $G Q(2)$. 
This equitable partition corresponds to an equitable partition of $\Gamma=N L_{2}(10)$, into two cells of sizes 40 and 60 .

\section{Concluding comments}

The presentation in this extended abstract is a tip of iceberg. Much more concrete information may be found in 28, while 27], subject of ongoing polishing and development, will reflect all detected computer data about the graphs from family $\mathfrak{F}$. We see also more potential to return again to construction of further models for graphs in $\mathfrak{F}$, in particular relying on non-automorphic EPs. The new attempts to achieve, at least in part, enumeration of all EPs of restricted size of cells for graphs on 77 and 100 vertices are also on agenda. The paper [16] as well as a number of private communications of M. Mačaj to author MK, create a background to attack in nearest future the problem of existence of new tfSRGs with relatively small parameters. The parameter set on 162 vertices seems to be first interesting and realistic target.

It should be mentioned that the results in [16], as well as in numerous private communications of M. Mačaj to the author MK, show that a putative new primitive triangle free strongly regular graph $\Gamma$ with prescribed parameters will have a relatively small automorphism group $A u t(\Gamma)$; very concrete restrictions on the cycle structure of elements in $A u t(\Gamma)$ are typically available. Transformation of those restrictions to the language of equitable partitions may be regarded as a reasonable starting platform for attempts to construct a new tfSRG.

Note, however, that many experts in AGT do not believe at all that a new tfSRG exists. Needless to say that this question creates a great challenge for the modern scientific community.

\section{Acknowledgments}

We thank Andy Woldar for ongoing collaboration in this project. We acknowledge Sven Reichard and Christian Pech for sharing with us their draft versions of COCO II. Ongoing communication with Martin Mačaj is very helpful and stimulative. We also are pleased to thank Peter Cameron and Jozef Širáň for interest expressed to the topic of our research.

\section{References}

[1] M. D. Atkinson. On rank 3 groups having $\lambda=0$. Canad. J. Math., 29(4):845-847, 1977.

[2] L. Babel, S. Baumann, and M. Luedecke. Stabcol: An efficient implementation of the weisfeilerleman algorithm. Technical Report TUM-M9611, Technical University Munich, 1996.

[3] L. Babel, I. V. Chuvaeva, M. Klin, and D. V. Pasechnik. Algebraic combinatorics in mathematical chemistry. methods and algorithms. ii. program implementation of the weisfeiler-leman algorithm. Technical Report TUM-M9701, Fakultät für Mathematik, TU Münc, 1997. http://www-lit.ma. tum.de/veroeff/html/960.68019.html

[4] C. T. Benson and N. E. Losey. On a graph of Hoffman and Singleton. J. Combinatorial Theory Ser. B, 11:67-79, 1971.

[5] A. E. Brouwer, A. M. Cohen, and A. Neumaier. Distance-regular graphs, volume 18 of Ergebnisse der Mathematik und ihrer Grenzgebiete (3) [Results in Mathematics and Related Areas (3)]. Springer-Verlag, Berlin, 1989.

[6] A.E. Brouwer. http://www.win.tue.nl/ aeb/graphs/ 
[7] Dragoš Cvetković, Peter Rowlinson, and Slobodan Simić. An introduction to the theory of graph spectra, volume 75 of London Mathematical Society Student Texts. Cambridge University Press, Cambridge, 2010.

[8] I. A. Faradžev and M. H. Klin. Computer package for computations with coherent configurations. In Proc. ISSAC, pages 219-223, Bonn, 1991. ACM Press.

[9] I. A. Faradžev, M. H. Klin, and M. E. Muzichuk. Cellular rings and groups of automorphisms of graphs. In Investigations in algebraic theory of combinatorial objects, volume 84 of Math. Appl. (Soviet Ser.), pages 1-152. Kluwer Acad. Publ., Dordrecht, 1994.

[10] The GAP Group. GAP - Groups, Algorithms, and Programming, Version 4.4.12, 2008.

[11] Chris Godsil and Gordon Royle. Algebraic graph theory, volume 207 of Graduate Texts in Mathematics. Springer-Verlag, New York, 2001.

[12] Paul R. Hafner. On the graphs of Hoffman-Singleton and Higman-Sims. Electron. J. Combin., 11(1):Research Paper 77, 33 pp. (electronic), 2004.

[13] M. Klin, C. Pech, and P.-H. Zieschang. Flag algebras of block designs. i. initial notions. steiner 2-designs and generalized quadrangles, November 1998.

[14] Mikhail Klin, Christian Pech, Sven Reichard, Andrew Woldar, and Matan Ziv-Av. Examples of computer experimentation in algebraic combinatorics. Ars Math. Contemp., 3(2):237-258, 2010.

[15] Mikhail H. Klin and Andrew J. Woldar. Dale Mesner, Higman \& Sims, and the strongly regular graph with parameters $(100,22,0,6)$. Bull. Inst. Combin. Appl., 63:13-35, 2011.

[16] Martin Mačaj and Jozef Širáň. Search for properties of the missing Moore graph. Linear Algebra Appl., 432(9):2381-2398, 2010.

[17] Spyros S. Magliveras. The subgroup structure of the Higman-Sims simple group. Bull. Amer. Math. Soc., 77:535-539, 1971.

[18] B. D. McKay. nauty user's guide (version 1.5), 1990.

[19] Dale Marsh Mesner. An investigation of certain combinatorial properties of partially balanced incomplete block experimental designs and association schemes, with a detailed study of designs of latin square and related types. ProQuest LLC, Ann Arbor, MI, 1956. Thesis (Ph.D.)-Michigan State University.

[20] Dale Marsh Mesner. Negative latin square designs. Mimeo notes 410, Institute of Statistics, UNC, NC, 1964.

[21] George Neil Robertson. Graphs minimal under girth, valency and connectivity constraints. ProQuest LLC, Ann Arbor, MI, 1969. Thesis (Ph.D.)-University of Waterloo (Canada).

[22] Martin Schönert et al. GAP - Groups, Algorithms, and Programming - version 3 release 4 patchlevel 4. Lehrstuhl D für Mathematik, Rheinisch Westfälische Technische Hochschule, Aachen, Germany, 1997.

[23] Leonard H. Soicher. GRAPE: a system for computing with graphs and groups. In Groups and computation (New Brunswick, NJ, 1991), volume 11 of DIMACS Ser. Discrete Math. Theoret. Comput. Sci., pages 287-291. Amer. Math. Soc., Providence, RI, 1993.

[24] Edwin R. van Dam. Three-class association schemes. J. Algebraic Combin., 10(1):69-107, 1999.

[25] B. Weisfeiler. On construction and identification of graphs. Number 558 in Lecture Notes in Math. Springer, Berlin, 1976.

[26] Robert Wilson, Peter Walsh, Jonathan Tripp, Ibrahim Suleiman, Richard Parker, Simon Norton, Simon Nickerson, Steve Linton, John Bray, and Rachel Abbott. Atlas of finite group representations - version 3. http://brauer.maths.qmul.ac.uk/Atlas/v3/.

[27] M. Ziv-Av. Protocols with computer data related to the investigation of known primitive tranglefree strongly regular graphs. http://www.math.bgu.ac.il/ zivav/math/

[28] M. Ziv-Av. Results of computer algebra calculations for triangle free strongly regular graphs. http://www.math.bgu.ac.il/ zivav/math/eqpart.pdf 


\section{A Data about embeddings of tfSRGs inside tfSRGs}

\begin{tabular}{|c|c|c|c|c|c|c|c|}
\hline & Quadrangle & edge & 2 edges & 3 & 4 & 5 & 6 \\
\hline Pentagon & 0 & 5 & 0 & 0 & 0 & 0 & 0 \\
\hline Petersen & 0 & 15 & 15 & 5 & 0 & 0 & 0 \\
\hline Clebsch & 40 & 40 & 60 & 40 & 10 & 0 & 0 \\
\hline $\mathrm{HoSi}$ & 0 & 175 & 7875 & 128625 & 845250 & 2170350 & 1817550 \\
\hline Gewirtz & 630 & 280 & 15120 & 245280 & 1370880 & 2603664 & 1643040 \\
\hline Mesner & 6930 & 616 & 55440 & 1330560 & 10589040 & 28961856 & 24641232 \\
\hline$N L_{2}(10)$ & 28875 & 1100 & 154000 & 5544000 & 67452000 & 301593600 & 477338400 \\
\hline & 7 edges & 8 & 9 & $\begin{array}{ll}10 \\
\end{array}$ & \begin{tabular}{|l|}
11 \\
\end{tabular} & & \\
\hline HoSi & 40150 & 15750 & 3500 & 350 & 0 & & \\
\hline Gewirtz & 104160 & 7560 & 1400 & 112 & 0 & & \\
\hline Mesner & 3664320 & 166320 & 30800 & 2464 & 0 & & \\
\hline$N L_{2}(10)$ & 258192000 & 14322000 & 924000 & 154000 & 11200 & & \\
\hline
\end{tabular}

Table 1: Number of imprimitive tfSRGs inside tfSRGs

\begin{tabular}{|c|c|c|c|c|c|c|c|c|c|}
\hline & \multicolumn{2}{|c|}{ Quadrangle } & & & 2 edges & 3 & 4 & 5 & 6 \\
\hline Pentagon & \multicolumn{2}{|c|}{0} & & 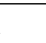 & 0 & 0 & 0 & 0 & 0 \\
\hline Petersen & \multicolumn{2}{|l|}{0} & & 1 & 1 & 1 & 0 & 0 & 0 \\
\hline Clebsch & \multicolumn{2}{|l|}{1} & & 1 & 1 & 1 & 1 & 0 & 0 \\
\hline HoSi & \multicolumn{2}{|l|}{0} & & 1 & 1 & 4 & 10 & 21 & 15 \\
\hline Gewirtz & \multicolumn{2}{|l|}{1} & & 1 & 2 & 9 & 30 & 48 & 36 \\
\hline Mesner & \multicolumn{2}{|l|}{1} & & 1 & 1 & 7 & 26 & 56 & 50 \\
\hline$N L_{2}(10)$ & \multicolumn{2}{|l|}{1} & & 1 & 1 & 2 & 7 & 14 & 17 \\
\hline & 7 edges & 8 & 9 & $\overline{\overline{10}}$ & 11 & & & & \\
\hline HoSi & 8 & 1 & 1 & 1 & 0 & & & & \\
\hline Gewirtz & 5 & 2 & 2 & 1 & 0 & & & & \\
\hline Mesner & 14 & 2 & 2 & 1 & 0 & & & & \\
\hline$N L_{2}(10)$ & 14 & 3 & 2 & 2 & 1 & & & & \\
\hline
\end{tabular}

Table 2: Number of orbits of imprimitive tfSRGs inside tfSRGs

\begin{tabular}{|c|c|c|c|c|c|c|c|}
\hline & Pentagon & Petersen & Clebsch & HoSi & Gewirtz & Mesner & $N L_{2}(10)$ \\
\hline Pentagon & 1 & 12 & 192 & 1260 & 8064 & 88704 & 443520 \\
\hline Petersen & & 1 & 16 & 525 & 13440 & 1921920 & 35481600 \\
\hline Clebsch & & & 1 & 0 & 0 & 0 & 924000 \\
\hline HoSi & & & & 1 & 0 & 0 & 704 \\
\hline Gewirtz & & & & & 1 & 22 & 1030 \\
\hline Mesner & & & & & & 1 & 100 \\
\hline$N L_{2}(10)$ & & & & & & & 1 \\
\hline
\end{tabular}

Table 3: Number of primitive tfSRGs inside tfSRGs 


\begin{tabular}{|c|c|c|c|c|c|c|c|}
\hline & Pentagon & Petersen & Clebsch & $\mathrm{HoSi}$ & Gewirtz & Mesner & $N L_{2}(10)$ \\
\hline Pentagon & 1 & 1 & 1 & 1 & 1 & 1 & 1 \\
\hline Petersen & & 1 & 1 & 1 & 1 & 9 & 5 \\
\hline Clebsch & & & 1 & 0 & 0 & 0 & 1 \\
\hline HoSi & & & & 1 & 0 & 0 & 1 \\
\hline Gewirtz & & & & & 1 & 1 & 1 \\
\hline Mesner & & & & & & 1 & 1 \\
\hline$N L_{2}(10)$ & & & & & & & 1 \\
\hline
\end{tabular}

Table 4: Number of orbits of primitive tfSRGs inside tfSRGs

\section{B Data about equitable partitions of primitive tfSRGs}

\begin{tabular}{|l|c|c|c|c|c|c|c|}
\hline & Pentagon & Petersen & Clebsch & HoSi & Gewirtz & Mesner & $N L_{2}(10)$ \\
\hline EP & 3 & 11 & 46 & 163 & & & \\
\hline Aut & 3 & 11 & 38 & 89 & 154 & 236 & 607 \\
\hline
\end{tabular}

Table 5: Number of orbits of equitable partitions and of automorphic equitable partitions for known tfSRGs.

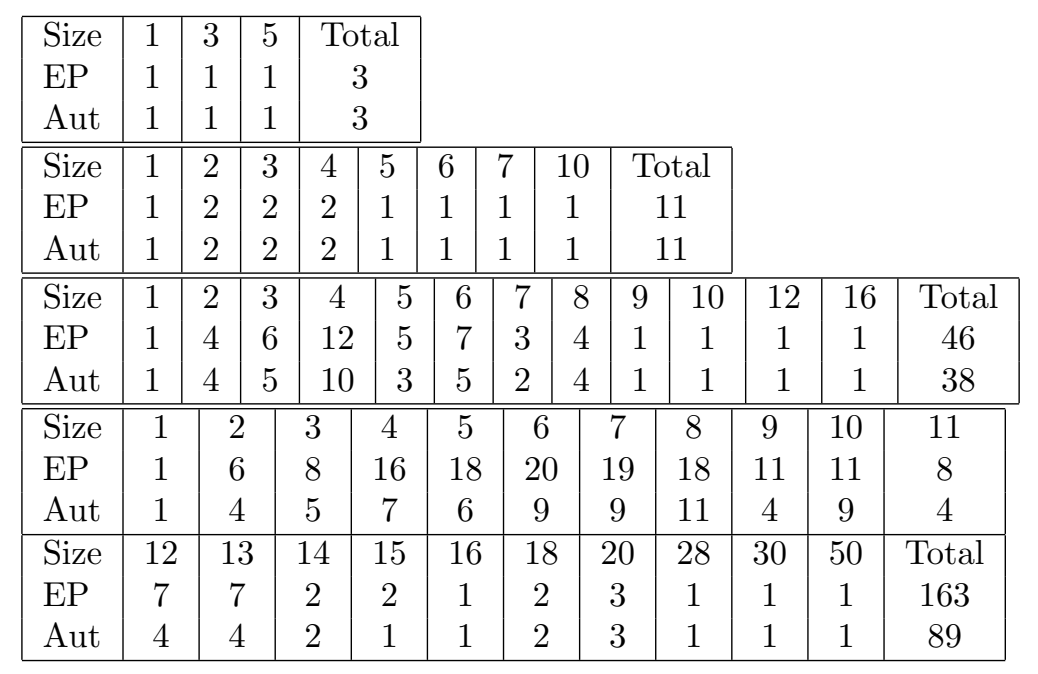

Table 6: Numbers of EPs and automorphic EPs by size of partition for four small graphs. 


\begin{tabular}{|c|c|c|c|c|c|c|c|c|c|c|c|c|c|c|c|}
\hline Size & 1 & 2 & 3 & 4 & 5 & 6 & 7 & 8 & 9 & 10 & 11 & 12 & 13 & \multicolumn{1}{|}{} \\
Aut & 1 & 5 & 9 & 12 & 12 & 14 & 15 & 16 & 14 & 11 & 7 & 7 & 6 & \\
\hline Size & 14 & 16 & 17 & 18 & 19 & 20 & 23 & 28 & 31 & 32 & 35 & 56 & Total & \\
Aut & 5 & 3 & 3 & 2 & 2 & 4 & 1 & 1 & 1 & 1 & 1 & 1 & 154 & 15 \\
\hline \hline Size & 1 & 2 & 3 & 4 & 5 & 6 & 7 & 8 & 9 & 10 & 11 & 12 & 13 & 14 & 15 \\
Aut & 1 & 3 & 5 & 8 & 11 & 10 & 20 & 14 & 19 & 12 & 18 & 12 & 16 & 9 & 14 \\
\hline Size & 16 & 17 & 18 & 19 & 20 & 21 & 23 & 25 & 29 & 33 & 41 & 45 & 49 & 77 & Total \\
Aut & 5 & 12 & 5 & 9 & 1 & 8 & 4 & 8 & 6 & 2 & 1 & 1 & 1 & 1 & 236 \\
\hline \hline Size & 1 & 2 & 3 & 4 & 5 & 6 & 7 & 8 & 9 & 10 & 11 & 12 & 13 & 14 & 15 \\
Aut & 1 & 6 & 15 & 21 & 28 & 29 & 31 & 42 & 34 & 35 & 37 & 49 & 30 & 31 & 27 \\
\hline Size & 16 & 17 & 18 & 19 & 20 & 21 & 22 & 23 & 24 & 25 & 26 & 27 & 28 & 29 & \\
Aut & 26 & 18 & 18 & 13 & 26 & 14 & 11 & 7 & 9 & 6 & 6 & 5 & 2 & 1 & \\
\hline Size & 30 & 31 & 32 & 33 & 34 & 35 & 39 & 40 & 45 & 50 & 53 & 60 & 65 & 100 & Total \\
Aut & 7 & 1 & 3 & 2 & 2 & 3 & 1 & 4 & 1 & 1 & 1 & 1 & 1 & 1 & 607 \\
\hline
\end{tabular}

Table 7: Numbers of automorphic EPs by size of partition for larger graphs. 